\title{
改进轻量级卷积神经网络的复杂场景口罩佩戴检测方法
}

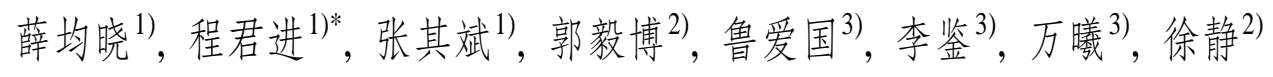 \\ 1) (郑州大学软件学院 郑州 450002) \\ 2) (郑州大学信息工程学院 郑州 450001) \\ 3) (中国船舶重工集团第 709 研究所 武汉 430070) \\ (jj.cheng@gs.zzu.edu.cn)
}

\begin{abstract}
摘 要: 针对复杂光照和人脸倾斜条件下口罩佩戴检测准确率低的问题, 提出一种利用轻量级卷积神经网络在复杂 环境下的口罩佩戴检测方法. 该方法利用难样本挖掘预训练学习更多的人脸特征, 结合多任务级联卷积神经网络先 判别是否有人脸信息, 对其进行精准的人脸定位; 在特征金字塔网络中添加注意力机制, 增强了人脸关键点信息的 权重, 利用轻量级神经网络将口罩佩戴检测视为简单的二分类问题进行判断. 在 TensorFlow 的环境下完成了数据训 练、数据预处理、与 AIZOO 方法对比实验, 收集建立了包含 816 张图片的数据集进行标注并训练; 在对数据进行预 处理操作时先将图片设定为固定大小以降低运算量, 提高检测速度, 再利用图像增强算法进行扭曲处理提高模型的 鲁棒性. 在此基础上, 利用 MTCNN 检测图片中的人脸并对其进行修正和归一化操作, 然后传人神经网络并利用已 经训练好的模型进行检测. 实验结果表明，在复杂光照和人脸倾斜等复杂条件下，文中方法的准确率分别达到 $83 \%$ 和 91\%, 可以有效地进行口罩佩戴检测.
\end{abstract}

关键词: 口罩佩戴检测; 目标定位; 目标分类; 轻量级卷积神经网络; 注意力机制

中图法分类号: TP391.41ＤOI: 10.3724/SP.J.1089.2021.18635

\section{Improved Efficient Convolutional Neural Networks for Complex Scene Mask-Wearing Detection}

\author{
Xue Junxiao ${ }^{1)}$, Cheng Junjin ${ }^{1{ }^{*}}$, Zhang Qibin ${ }^{1)}$, Guo Yibo ${ }^{2)}$, Lu Aiguo ${ }^{3)}$, Li Jian ${ }^{3)}$, Wan Xi ${ }^{3)}$, and Xu Jing ${ }^{2)}$ \\ 1) (School of Software Engineering, Zhengzhou University, Zhengzhou 450002) \\ ${ }^{2)}$ (School of Information Engineering, Zhengzhou University, Zhengzhou 450001) \\ ${ }^{3)}$ (No.709 Research Institute of China Shipbuilding Industry Corporation, Wuhan 430070)
}

\begin{abstract}
To solve the problem about low accuracy of mask wear detection under complex lighting and face lean conditions, a method of mask wear detection under intricate environment using efficient convolutional neural network is proposed, which uses pre-training such as hard negative mining to learn more samples of face feature, utilize multi-task convolutional neural networks (MTCNN) to estimate the possibility of face information, and get accurate face location. With attention mechanism in feature pyramid network, enhancing the weight of key points on human face, employing efficient neural network detection will be wore on mask-wearing detection as a simple binary classification problem. Under the environment of TensorFlow platform, not only data training, data preprocessing, but also the contrast experiment with AIZOO method
\end{abstract}

收稿日期：2020-09-01; 修回日期：2020-11-14. 基金项目：河南省科技厅应急攻关项目(201100312000); 河南省高等学校青年骨 干教师培养计划(22020GGJS014). 薛均晓(1982一), 男, 博士, 副教授, 硕士生导师, CCF 会员, 主要研究方向为人工智能、虚拟现实; 程君进(1994-), 女, 硕士研究生, 论文通讯作者, 主要研究方向为计算机视觉; 张其斌(1996一), 男, 硕士研究生, 主要研究方向为 计算机视觉; 郭毅博(1987一), 男, 博士, 讲师, 硕士生导师, 主要研究方向为机器学习; 鲁爱国(1979一), 男, 学士, 研究员, 主要研 究方向为图形图像处理; 李鉴(1969-), 男, 学士, 研究员, 主要研究方向为机器视觉; 万㬢(1981一), 男, 学士, 高级工程师, 主要 研究方向为机器学习; 徐静(1983一), 女, 学士, 讲师, 主要研究方向为数字图像处理. 
are completed. A data set containing with 816 images is collected, marked and trained. During the data preprocessing, images are set as fixed size to reduce the amount of computation and promote the detection speed. Then, image enhancement algorithm is used to conduct distortion processing to improve the robustness of this model. On this basis, MTCNN is used to detect the face information in pictures, modify and normalize all data, then put them into neural network and the trained model to detection. The experimental results show that under complex conditions such as complex lighting and face tilt, the accuracy can reach 83\% and 91\% respectively, which means can accurately detect whether wearing a mask.

Key words: mask-wearing detection; object location; object classification; MobileNet; attention mechanism

专家研究发现, 新型冠状病毒属于急性呼吸 道传染病, 主要通过呼吸道传播, 在人和人的接触 过程中佩戴口罩可以有效地降低感染风险。所以 在疫情并未完全控制住的情况下, 患者佩戴口罩 可以降低传播风险, 避免出现一传多的现象; 医护 人员佩戴口罩等防护用具可以有效地避免在治疗 患者的过程中自己被传染, 而未感染者佩戴口罩 可以降低自己被感染的风险.

据有关数据统计, 现仍有一定比例的行人暴 露在易感环境中, 甚至还有部分行人口罩佩戴不 规范. 由于未感染者数量庞大, 且未感染者中不排 除无症状感染者和未检测感染者的存在, 完全交 由人力, 以人盯人的方式去监督检测不仅会增加 被感染的风险, 也受到人的精力和视力范围影响, 存在疏漏的可能.

基于这一现实情况, 本文提出使用机器学习 的方法实现口罩佩戴检测，不仅可以节省大量人 力物力, 减轻工作人员的负担, 也明显提高了检查 效率，扩大了监察范围，具有广阔的应用前景. 然 而，口罩佩戴检测基于人脸检测、目标分类技术发 展而来，相比于简单的人脸检测，口罩佩戴检测需 要解决目标遮挡严重问题. 与一般的目标分类问 题不同, 口罩佩戴检测问题需要在包括侧脸、阴 影、不同光照情况下准确地分辨出遮挡物是否为口 罩，如何解决这些问题并提高口罩佩戴检测技术 的准确度是当前面临的巨大挑战.

本文将口罩佩戴识别问题转化为简单的图片 二分类目标, 在人脸检测时, 利用难样本挖掘算法 提取出在侧脸、阴影、光照强度不充足等特殊情况 下的样本, 利用多任务卷积神经网络 (multi-task convolutional neural networks, MTCNN $)^{[1]}$ 对其进行 人脸定位, 提取定位结果. 将结果输人轻量级卷积 神经网络(efficient convolutional neural networks, MobileNet), 将定位结果进行二分类判断, 即 mask
和 nomask.

MTCNN 是一个基于级联卷积神经网络 ${ }^{[2]}$ 的人 脸检测和人脸对齐神经网络, 其总体可分为 3 层网 络结构: P-Net(proposal network), R-Net(refine network)和 O-Net(output network). 通过增加网络深 度、减少滤波器, MTCNN 在减少计算数据量的同 时提高其表征提取能力. 利用多任务联合训练, 在 检测人脸区域时获得人脸关键点的检测信息，并 将其融合，从而在很大程度上提高了人脸检测的 精度. 在训练样本时, 结合难样本挖掘技术对样本 进行梯度回传，网络收敛速度提升.

本文提出一种结合难样本挖掘的方法, 将 MTCNN 人脸检测方法的人脸定位结果作为轻量 级神经网络的人脸分类基础, 用于口罩佩戴识别 的检测应用中; 利用难样本挖掘学习更多的有效 特征获取准确的人脸信息, 结合注意力机制突出 人脸关键点特征, 可以通过关键点提高判断人脸 位置的精确度; 在光线暗、画面清晰度低、人脸遮 挡/倾斜/侧脸等面部检测不完整的复杂环境下都可 准确检测, 且在背景环境复杂和人脸遮挡较多的 条件下，检测精度分别达到 83\%和 91\%。

\section{1 口罩佩戴检测算法}

基于深度卷积神经网络 ${ }^{[3-4]}$ 的人脸检测算法, 多数都是基于深度学习目标检测算法进行的改进, 或者说是把通用的目标检测模型, 为适应人脸检 测任务而进行的特定配置. 在众多的目标检测模 型中, 人脸检测算法最常用的是单级多目标检测 算法(single shot multibox detector, SSD $)^{[5]}$, 文献 [6-8]和 SSH 模型 ${ }^{[9]}, S^{3} F D$ 模型 ${ }^{[10]}$ 及 RetinaFace 算 法 ${ }^{[11]}$ 都是受 SSD 算法的启发, 或者基于 SSD 进行 的任务定制化改进, 如将定位层提到更靠前的位 置、anchor 大小调整、anchor 标签分配规则的调整 
以及在 SSD 基础上加人特征金字塔网络等.

新冠肺炎疫情暴发后，一些大小型企业和实 验室等工作人员都投人口罩佩戴检测方法开发的 工作中.

疫情暴发后，阿里云视觉智能开放平台的人 脸口罩检测算法上线, 将人脸识别和口罩检测通 过应用程序接口(application programming interface, API)对接. 首先使用人脸定位功能识别普通人群 图片获取相应的人脸坐标，根据返回的对应字段 获取 1 组或多组人脸具体的坐标组合, 对出现在画 面中的所有人脸进行定位; 之后通过其对象存储 服务的图片处理能力对获取到的定位进行剪切, 当获取到裁切后图片的网络地址后, 将预处理过 的图片地址作为参数传人口罩检测接口; 最后利 用视觉智能开放平台的 “人脸口罩检测” 能力，完 成检测接口的调用并获取接口返回值，根据检测 的结果来触发后续的处理逻辑.

2018 年, 百度公司凭借全新的人脸检测深度 学习算法 PyramidBox ${ }^{[12]}$ 在 2 个常用人脸检测基准 FDDB 和 WIDER FACE ${ }^{[13]}$ 上的非凡表现, 刷新了 当年最好成绩. 疫情暴发后, 百度在 PyramidBox 算法的基础上进行改进, 实现了口罩佩戴检测功 能, 借助一种新的语境辅助网络框架, 充分利用上 下文的语境信号, 网络在学习人脸特征时, 使用半 监督解决方案生成与面部相关的语境部分的近似 标签, 使得能学习口罩、头部和身体等语境部分的 特征, 同时设计了低层次特征金字塔网络, 更好地 融合高级语义特征和口罩特征; 最后将上下文的 语境信号引人网络, 以增加网络的性能, 提高输出 结果的精度.

在基于特征融合和分割监督的人脸检测(face detection with feature fusion and segmentation supervision, $\mathrm{DF}^{2} \mathrm{~S}^{2}$ )方法 ${ }^{[14]}$ 基础上, 滴滴出行科技有 限公司结合人脸属性识别算法提出口罩佩戴检测 方法. 为了适用于不同光照、人脸姿态变化和测 量距离不等的条件，该方法通过扩展人脸区域和 对口罩位置采用注意力机制的算法，同时利用损 失函数权重策略和数据增强等方法解决白天/夜 晚戴口罩数据不均衡问题以及戴口罩类型多样化 问题, 能在各类场景图像中对人脸进行口罩佩戴 情况检测.

牛作东等 ${ }^{[15]}$ 提出改进 RetinaFace 的自然场景
口罩佩戴检测算法，其是在原来的人脸检测基础 上通过改进分类损失函数实现了人脸口罩佩戴检 测任务. 为了加快训练速度, 将人脸关键点三维分 析等无关检测取消, 并且改进金字塔注意力机制 ${ }^{[16]}$ 和自注意力机制 ${ }^{[17]}$, 达到了增强特征和利用上下 文关系提高特征图描述能力的目的, 最终可以在 自然场景下有效地检测口罩佩戴情况.

AIZOO $^{\circledR}$ 也提出一种基于 Faster R-CNN ${ }^{[18]}$ 和 YOLOV $^{[19]}$ 的目标检测方法, 实现口罩佩戴检测 任务, 并取得了不错的成绩. 该方法的基础网络在 目标检测任务中已经非常成熟且精确度高, 但是 在针对复杂光照、侧脸或面部特征遮挡面积较大的 情况下，检测精度还有待提高.

上述方法都是直接利用深度网络对人脸进行 检测, 判断是否佩戴了口罩, 在人脸识别时, 光 照、遮挡等因素会在很大程度上影响判断结果, 增 加神经网络计算的工作量. 唐振超 ${ }^{\circledR}$ 提出的人脸口 罩检测方法将口罩佩戴检测方法分为 2 步：首先使 用 MTCNN 进行人脸定位，获取人脸信息，对获取 到的人脸信息进行再处理; 然后将其输人轻量级 神经网络中实现口罩佩戴检测分类判断. 将人脸 口罩佩戴检测工作分为人脸检测和口罩佩戴分类 2 步, 可以有效地简化网络结构, 减少神经网络计 算量, 避免了检测失误; 但是, 该方法只适用于光 照合理、人脸无倾斜等理想的条件下，一旦遇到图 像模糊、光照强度变化、人脸倾斜等情况，口罩佩 戴检测的精度会大大降低. 此外, 普通的目标检测 算法无法正确地检测人脸, 也会直接影响口罩佩 戴检测的效果.

针对这些情况, 本文结合难样本挖掘 ${ }^{[20]}$, 实 现了在移动端利用 MTCNN 算法来定位人脸位置、 再结合 MobileNet ${ }^{[21]}$ 进行口罩佩戴检测的目标. 实 验结果表明, 本文方法可以有效地提高人脸口罩 佩戴检测的准确率.

\section{2 本文方法}

本文利用 MobileNet 根据已有的数据集进行 训练. 在进行目标检测的训练过程中, 先用初始的 正负样本 (一般是正样本 + 与正样本同规模负样本 的一个子集)训练分类器, 然后用训练出的分类器 对样本进行分类; 提取的特征中除了对结果有积

(1) https://github.com/aky15/AIZOO_torch

(2) https://github.com/bubbliiiing/mask-recognize 
极影响的特征外, 可能还会包括很多对检测结果 造成误差的特征. 将这些特征命名为难样本, 将其 中错误分类的那些样本(即难样本)放人负样本集 合, 再继续训练分类器, 多次训练, 直至达到停止 条件(如分类器性能不再提升), 可以解决目标检测 低召回率和样本不平衡问题. 经过难样本挖掘训 练之后可以得到分类模型, 测试时输人一张带有 人脸的图片, 经过卷积处理得到特征图; 将获取的 特征图经过 MTCNN 输出人脸框的坐标信息和 5 个关键点坐标，再将人脸区域从原图中截取下来; 通过注意力机制提高截取到的人脸信息置信度, 以确保获取到的是完整有效的人脸信息，然后将 截取的人脸区域通过 MobileNet 模型预测，最终返 回 mask 或 nomask 的实验结果.

\subsection{MTCNN 人脸检测的工作原理}

人脸检测即对输人网络中可能存在人脸的图 片进行检测，对检测到的人脸用矩形框定位标注. MTCNN 实现了人脸对齐功能, 即检测人脸中的 5 个关键点, 包括眼睛、鼻子和嘴角的位置信息, 每 个关键点用二维坐标信息表示.

MTCNN 参考级联卷积神经网络 ${ }^{[22]}$ 的思想, 将总体网络结构分为 P-Net, R-Net 和 O-Net 这 3 层 子网络, 每层网络都获得既定的信息, 输人下一层 网络可以学习到更多、更准确的信息, 在训练网络 参数的同时采用多任务的形式，构成了人脸检测 由笼统到精确的一种算法结构. 这种结构减少了 时空计算, 人脸检测速度更快且检测更准确.

MTCNN 的人脸检测算法由 3 级卷积神经网络 P-Net, R-Net 和 O-Net 组合构成. 在图像输人级联 网络之前, 需要先根据每级网络输人图片的大小 对原始图片裁剪，构建图像金字塔，以适应不同大 小的人脸并进行检测 ${ }^{[23]}$. 在级联网络检测过程中, 用到了边界框回归和非极大值抑制(non maximum suppression, NMS)算法 ${ }^{[24]}$, 选择最佳的人脸候选 框, 以保证输出人脸候选框和人脸关键点坐标的 信息更精确，从而提高口罩佩戴检测的检测精度.

\subsection{MTCNN 结构}

MTCNN 通过级联的方式，采用候选框和分类 器的思想进行快速、高效的人脸检测. 其中, 图片 先经过 P-Net, 快速判断是否存在人脸信息, 并生 成大量的候选框; 经过 R-Net 过滤, 将 P-Net 生成 的无效候选框篎除，生成高精度候选框; 最后将高 精度候选框输人 O-Net, 经过第 2 次过滤，生成最 终边界框与人脸关键点 ${ }^{[25]}$.
P-Net 的作用是判断输人图像样本中是否存在 人脸, 并给出人脸区域矩形框回归和关键点的初 步判定. 如图 1 中 P-Net 结构所示, 其基本构造是 一个全连接网络, 将通过图像金字塔进行尺度变 换的图片特征输人 3 个卷积层之后, P-Net 会通过 人脸分类器判断该区域是否存在人脸，同时使用 边框回归和 NMS 算法对不合格的窗口进行过滤, 最后将面部关键点定位器输出的多张可能存在人 脸的区域结果输人 R-Net 进行下一步处理 ${ }^{[26]}$.

R-Net 的作用是给出人脸框的精确位置信息. 由于在 R-Net 层检测到的人脸框可能存在侧脸或 光线较暗等因素, 因此需要输出当前框位置相对 完美的人脸框位置的偏移. 如图 1 中 R-Net 结构所 示, 与 P-Net 相比, R-Net 增加了一个全连接层, 篮 选人脸预测框的约束条件会更加严格; R-Net 会从 由 P-Net 输人的所有预测框中滤除掉大量效果较 差的预测框, 因此得到的候选框位置精度要优于 P-Net. 得到较精确的候选框之后, 输出高精度人 脸区域候选框到 O-Net，用于更精细的检测.

如图 1 中 O-Net 结构所示. O-Net 比 R-Net 多 了一个卷积层, 是一个更复杂的卷积网络. 与 R-Net 的功能相比, O-Net 保留了更多的图像特征, 通过更多的监督识别面部的区域, 所有特征信息 经过网络结构的全连接层保留; 同时进行人脸判 别、边框回归和特征定位, 最终输出人脸区域的边 界框坐标信息、score 以及人脸区域的 5 个关键点 坐标 ${ }^{[27]}, 5$ 个关键点分别是双眼和鼻子以及嘴角.

\section{3 激活函数}

在训练人工神经网络时，激活函数可以有效 地提高学习神经网络、理解非常复杂和非线性的函 数的效率, 对神经网络的输出值进行拟合作用, 增 强神经网络的表达能力. 常用的非线性激活函数 有 ReLU(rectified linear unit), 该函数将矩阵内所 有负值都设为零, 其余的值不变, 可以加快收玫速 度，解决梯度消失问题，函数定义为

$$
f(x)=\left\{\begin{array}{l}
0, x<0 \\
x, x \geqslant 0
\end{array} .\right.
$$

本文使用带参数的 ReLU 激活(parametric rectified linear unit, PReLU) 函数 ${ }^{[28]}$. 相比 ReLU 函数直接 过滤掉负值的计算方法, 该函数对负值进行了添加 参数的操作, 但增加的参数量是极少的, 对计算速 度和拟合过程的影响较小. ReLU 函数和 PReLU 函数 的区别如图 2 所示. 如果 $a_{i}=0$, PReLU 函数和 ReLU 函数结果相同, 其中, $i$ 表示不同的通道. 


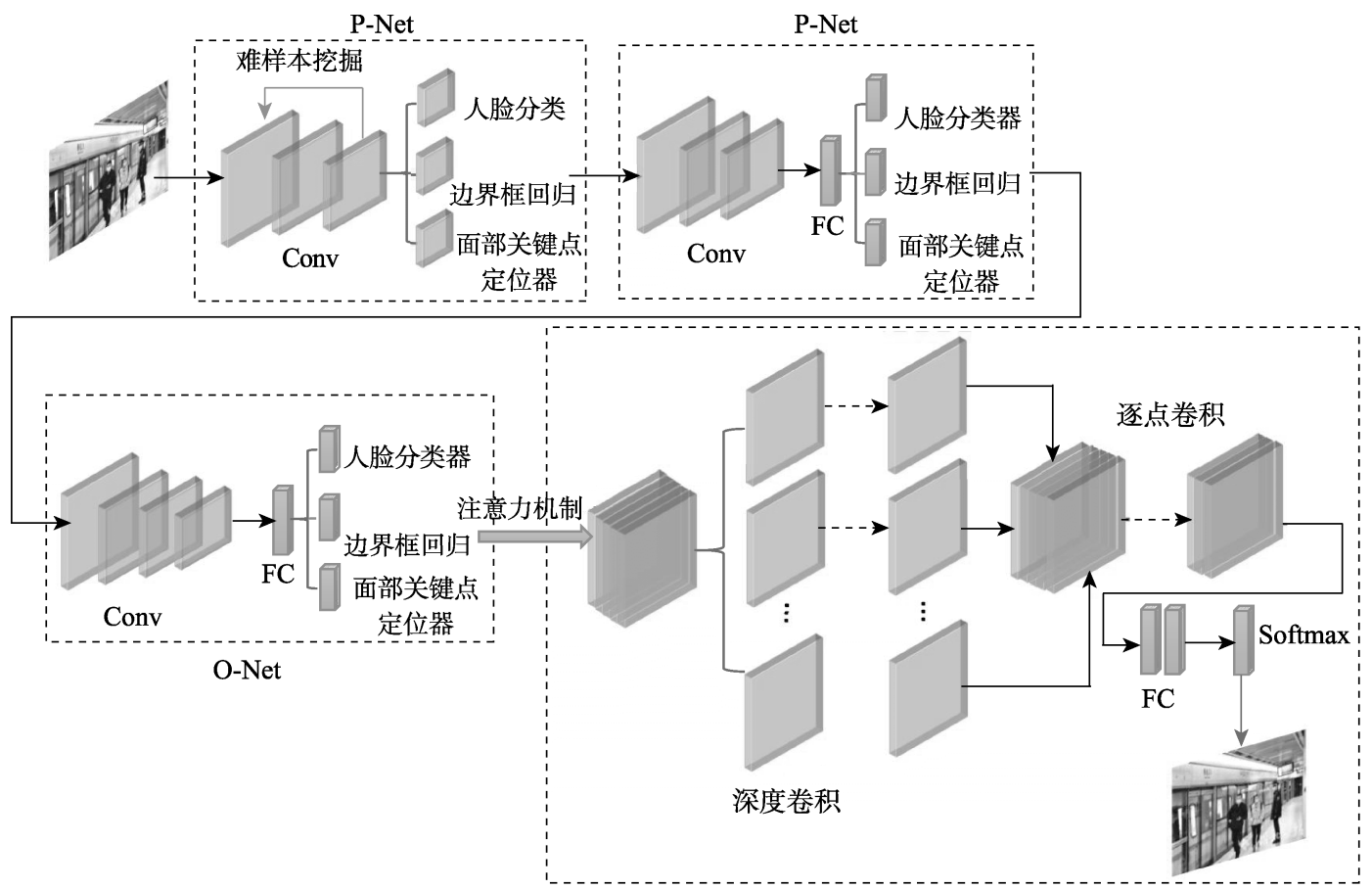

图 1 改进 MobileNet 的口罩检测方法结构

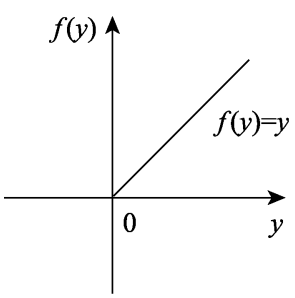

a. $\operatorname{ReLU}(y)=\left\{\begin{array}{l}y, y>0 \\ 0, y \leqslant 0\end{array}\right.$

图 2 ReLU 和 PReLU 对比图

\subsection{NMS}

在人脸检测获得目标候选框时, 每个候选框 都会有一个相应的分值. NMS 算法的作用就是与 原图的 ground-truth 目标框作交并比(intersection over union, IoU)运算, 根据 IoU 值过滤掉同一个目 标 IoU 值相对较小的候选框, 将 IoU 值最大的候选 框保留, 即目标框.

本文算法在人脸数据中通过多尺度变换生成 多个不同尺寸的人脸检测矩形框，同时检测矩形 框中的图像特征. 卷积网络会对检测到的人脸生 成多个不同比例的预测候选框，每个预测候选框 都有一个置信度评分，但并不是所有的候选框都 符合要求, NMS 的作用就是在多个预测候选框中 篮选出分值最高的候选框作为目标框. 如图 3 所 示, 图 3a 为包含多个预测候选框的结果图; 图 3b 为经过 NMS 之后, 生成目标得分最高的人脸候选 框结果图.

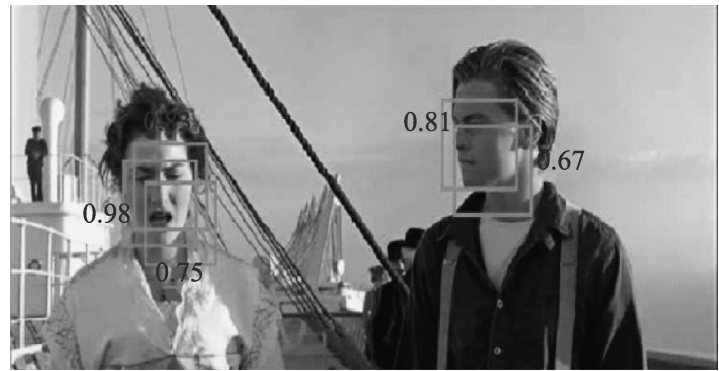

a. 目标候选框

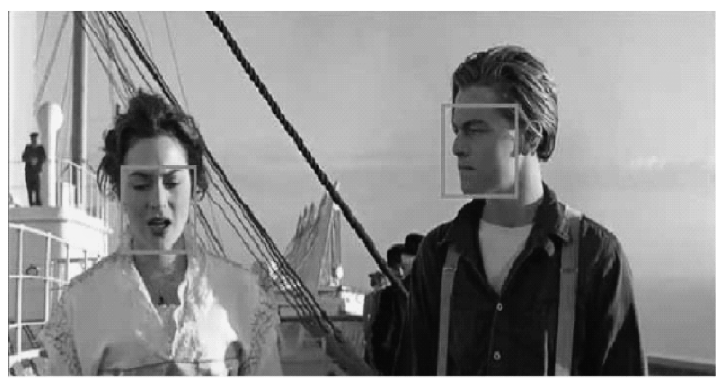

b. 目标框

图 3 NMS 效果图

\subsection{MobileNet 结构}

为了在移动设备和嵌人式应用上实现目标分 类、检测等图像处理功能, Google 在 2017 年提出 了 MobileNet, 其核心思想是使用深度可分离卷积 取代常规的卷积层, 即分别收集通道和空间区域 特征. 深度可分离卷积由深度卷积和逐点卷积组 成 ${ }^{[29]}$, 在深度卷积过程中, 卷积核只收集单个通 道内部的空间特征信息，通道之间信息不共享，而 
逐点卷积通过收集每个点的特征信息, 联合深度 卷积收集到的多个通道空间信息.

把普通卷积分解为一个深度卷积和一个逐点 卷积, 分别起到滤波和线性组合的作用，同时减少 参数量和计算量, 这种分离方式可明显降低计算 量. 本文采用 MobileNet 实现口罩佩戴的预测, 可 以实时进行预测结果的显示.

\section{3 实验及结果分析}

本文在训练好的模型基础上进行实验，实验 环境为 i5-6300 HQ CPU, 12.00 GB RAM, GTX960 $\mathrm{m} 2 \mathrm{G}$ 显卡; 软件环境为 Windows 10 操作系统, 集 成开发环境为 PyCharm.

\section{1 数据集}

由于没有公开大量的复杂场景下口罩佩戴检 测数据集, 因此在实验时采用自行制作的数据集. 在此数据集中共收集了 816 张图片, 对其进行手工 标注和模型训练与预测, 将其分为 mask 和 nomask, 部分数据集中的图片如图 4 所示.

\section{2 损失函数}

在训练过程中, 利用 MTCNN 模型的 3 级网 络, 分别进行难样本挖掘、面部/非面部分类、边界 框回归、面部地标定位和注意力机制计算.
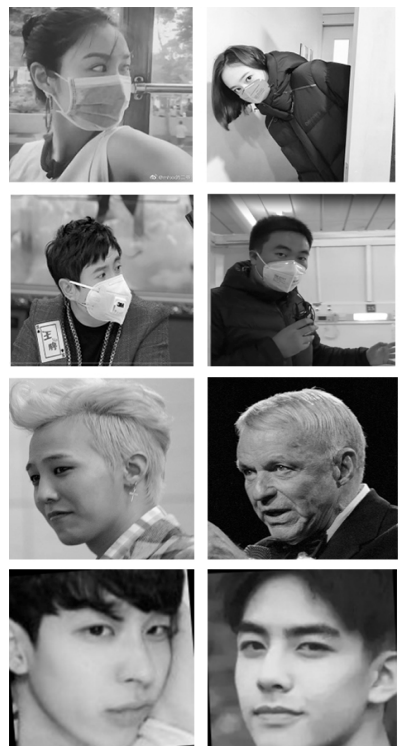
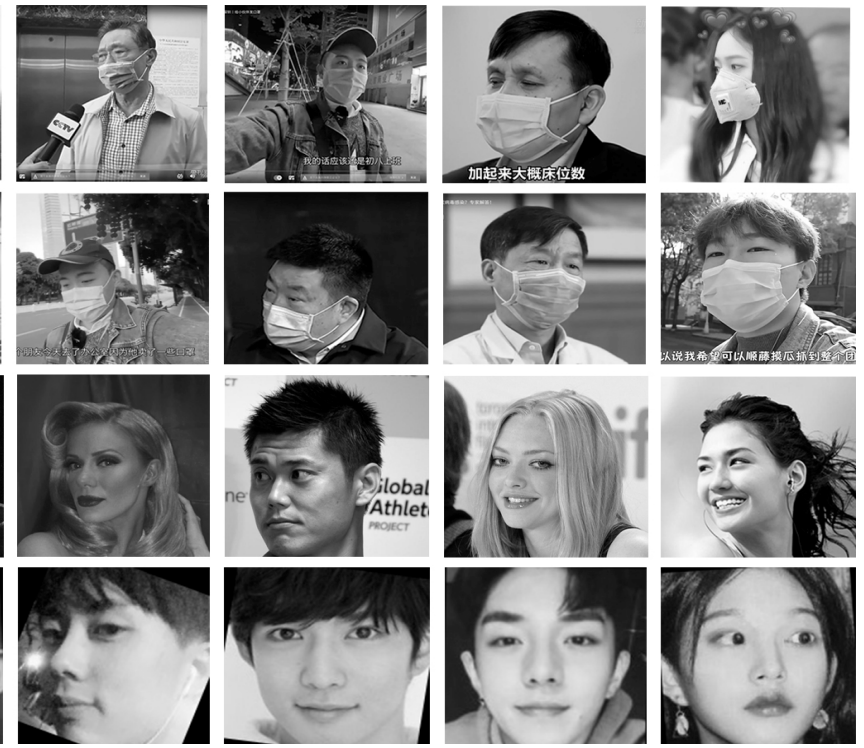

图 4 部分数据集图片

\section{(1) 难样本挖掘}

与原始分类器训练后进行传统难样本挖掘不 同，本文方法在面部/非面部分类任务中在线难样 本挖掘, 以适应训练过程. 特别是在每个小批量 中，首先对来自所有样本的前向传播中计算的损 失排序, 并选择其中前 70 作为难样本; 然后只计 算后向传播中难样本的梯度. 这意味着在训练期 间对于加强探测器意义不大的简单样本忽略不计.

(2) 面部分类

该实验阶段将学习目标制定为二分类问题， 首先对目标进行是否存在人脸的检测，即人脸非 人脸存在分类. 对于每个样本 $x_{i}$, 使用交叉熵损 失函数 $L_{i}^{\mathrm{det}}=-\left(y_{i}^{\mathrm{det}} \lg \left(p_{i}\right)+\left(1-y_{i}^{\mathrm{det}}\right)\left(1-\lg \left(p_{i}\right)\right)\right)$. 其 中, $p_{i}$ 表示网络产生的概率, 表明样本是网络产生 的概率; $y_{i}^{\mathrm{det}} \in\{0,1\}$ 表示地面实况标签.
（3）边界框回归

人脸检测时不仅要判断是否存在人脸信息, 更希望标记出的人脸区域尽可能准确, 常见的方 法是使用边界框回归算法实现. 边界框回归采用 欧几里得损失函数 $L_{i}^{\mathrm{box}}=\left\|\hat{y}_{i}^{\mathrm{box}}-y_{i}^{\mathrm{box}}\right\|_{2}^{2}$. 其中, $\hat{y}_{i}^{\mathrm{box}}$ 为从网络获得的回归目标; $y_{i}^{\mathrm{box}}$ 为地面实况 坐标, 实况坐标分别包括左上角坐标、高度和宽度 信息，因此， $y_{i}^{\text {box }} \in \mathbb{R}^{4}$.

(4) 面部地标定位

类似于边界框回归任务，面部地标检测被公 式化为回归问题, 使用最小化欧几里得损失 $L_{i}^{\text {landmark }}=\left\|\hat{y}_{i}^{\text {landmark }}-y_{i}^{\text {landmark }}\right\|_{2}^{2}$. 其中, $\hat{y}_{i}^{\text {landmark }}$ 表

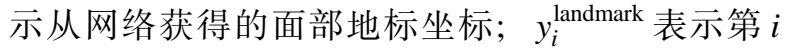
个样本的地面实况坐标, 由 5 个关键点坐标组成 
(左右眼、鼻子、嘴角), 每个点用二维坐标表示, 因 此, $y_{i}^{\text {landmark }} \in \mathbb{R}^{10}$.

(5) 注意力机制

在神经网络中, 能够硬性选择输入的某些部 分或者给输人的不同部分分配不同的权重. 深度 学习与视觉注意力机制结合的工作大多数集中于 使用掩码形成注意力机制, 掩码的原理是通过另 一层新的权重将图片数据中关键的特征标识出, 通过学习训练让深度神经网络学习到每张新图片 中需要关注的区域, 也就形成了注意力. 现实情况 下人脸可能存在各种遮挡和光线影响, 导致无法 正确地判断是否为人脸以及是否佩戴口罩, 使用 注意力机制可以使网络更好地学习关键点信息, 以确保更准确地检测到人脸特征。

MTCNN 实现的人脸检测和关键点定位效果 如图 5 所示.

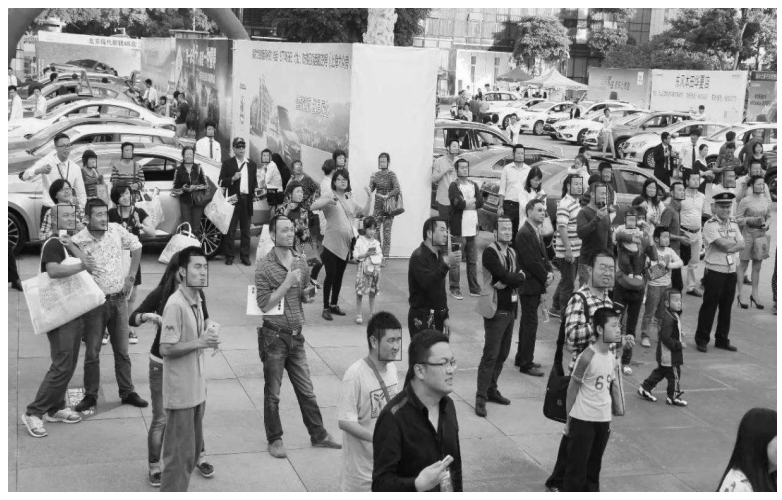

图 5 MTCNN 人脸检测结果

\section{3 改进 MobileNet 的口罩佩戴检测}

MobileNet 的核心在于其深度可分离卷积网 络, 通过引人深度卷积和逐点卷积多任务工作方 法, 极大程度地降低了参数数量与运算量, 提高 了训练速度, 并且对分类结果精度的影响可忽略 不计.

深度可分离卷积由深度卷积和逐点卷积构成. 与普通卷积将卷积核用于所有输人通道上不同, 深度卷积针对不同通道采用不同的卷积核，即通 道和卷积核之间一一对应. 深度卷积层后连接了 批量归一化网络层 (batch normalization, BN) 和 ReLU 激活层, BN 层将数据归一化后能够加速网络 收玫，控制数据过拟合，解决梯度消失问题.

与普通卷积相比, 深度可分离卷积减少了大 量的计算数据和参数数量, 使现有的卷积网络可 以在更多的移动平台使用. 设定卷积输人特征图 $F$ 的大小为 $D_{F} \times D_{F} \times M$, 而输出特征图 $G$ 的大小为
$D_{G} \times D_{G} \times N$, 其中, $D$ 表示特征图的宽和高, $M$ 和 $N$ 分别表示输人通道数和输出通道数. 普通卷积的 卷积核学习所有通道上的空间特征, 卷积核 $K$ 包 含 $D_{K} \times D_{K} \times M \times N$ 个参数, 设步长为 1 . 普通卷积 输出可表示为 $G_{k, l, n}=\sum_{i, j, m} K_{i, j, m, n} F_{k+i-1, l+j-1, m}$. 其中, 普通卷积计算量为 $D_{K} \times D_{K} \times M \times N \times D_{F} \times D_{F}$.

深度卷积的每个卷积只学习一个通道的空间 特征, 则深度卷积输出式为

$$
\hat{G}_{k, l, n}=\sum_{i, j, m} \hat{K}_{i, j, m, n} F_{k+i-1, l+j-1, m} .
$$

其中, $\hat{K}$ 代表卷积核包含的参数量 $D_{K} \times D_{K} \times M$, $\hat{K}$ 中第 $m$ 个滤波器被应用到 $F$ 中的第 $m$ 张特征图 中, 产生 $G$ 中第 $m$ 张特征图.

深度卷积的计算量为 $D_{K} \times D_{K} \times M \times D_{F} \times D_{F}$, 在深度卷积操作中, 每个卷积学习单通道的空间 特征, 这些特征都是分离的, 无法整合得到完整的 信息, 逐点卷积的功能就是通过收集每个点的特 征信息，使用卷积核尺寸为 $1 \times 1$ 的卷积层联合深度 卷积收集到的多个通道空间信息. 逐点卷积层后 的 BN 层和 ReLU 层增加了模型的非线性变化, 从 而增强模型的泛化能力.

因此, 深度可分离卷积的总计算量可以表示 为 $D_{K} \times D_{K} \times M \times D_{F} \times D_{F}+N \times M \times D_{F} \times D_{F}$, 对普 通卷积进行深度卷积和逐点卷积拆分, 相当于将 普通卷积的计算量压缩为

$$
\begin{aligned}
& \frac{D_{K} \times D_{K} \times M \times D_{F} \times D_{F}+N \times M \times D_{F} \times D_{F}}{D_{K} \times D_{K} \times M \times N \times D_{F} \times D_{F}}= \\
& \frac{1}{N}+\frac{1}{D_{K}^{2}} .
\end{aligned}
$$

\section{4 模型训练设置}

本文的实验环境配置参考文献 [30]，模型训 练、验证和测试均在 Google 的 TensorFlow 2.0 平 台上进行, 训练集和测试集随机分配. 其中, 训练 集约 730 张图片, 每轮训练完通过置乱的方式将数 据打乱. 为了提高训练效率, 使用 MobileNet 提供 的预训练权重. 实验过程中, 将学习率设置为 0.001, 采用小批量梯度下降法, 根据计算机的硬 件情况一次输人 4 张图片进行训练, 训练前将所有 训练图片像素调整为 $160 \times 160$, 以保证输人图片 的统一性.

采用交叉熵损失函数和 Adam 优化算法计算 每个参数的自适应学习率. 由于本文数据量较少, 模型相对简单，采用早停算法防止过拟合.

如图 6 所示, 在准确率趋于平稳之前, 模型在 
测试集和训练集的准确率成正比. 如果超过一定 训练步数后模型在训练集上的准确率还在上升, 但是在测试集上的准确率却不再变化, 模型就可 能存在过拟合现象. 因此, 通过早停算法检测训练 模型在测试集上的误差, 在训练的过程中记录目 前为止最好的验证准确率，当连续 10 次迭代都没 有达到最佳准确率时, 则认为准确率不再有提升, 此时停止迭代.

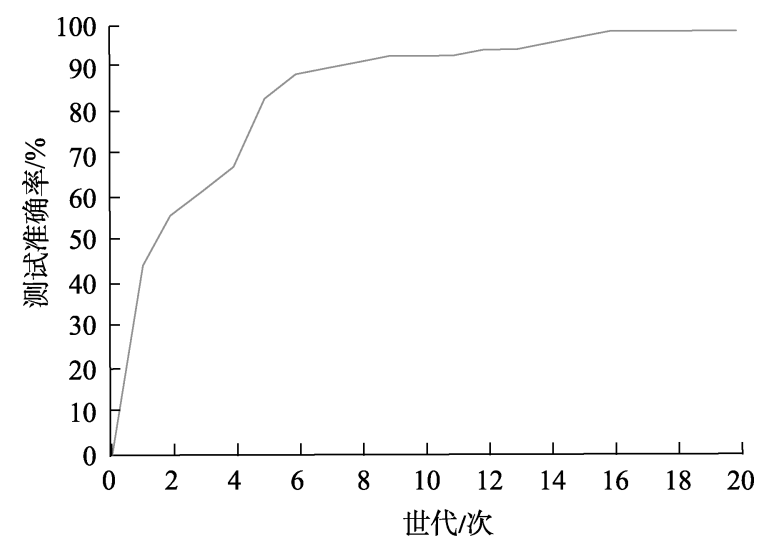

图 6 训练准确度变化曲线

\section{5 实验结果对比}

与其他模型在多人脸目标、人脸倾斜、侧脸、 光照影响条件下的检测结果相比, 本文方法都取 得了较好的成绩. 本文方法与 AIZOO 方法的人脸 口罩检测网络模型对比的实验结果如图 7 所示. 可 以看出，在 MTCNN 和 MobileNet 的加持下，本文 方法结合难样本挖掘和注意力机制实现了口罩佩 戴检测，对是否佩戴口罩做出了正确的判断; 在人 脸倾斜/侧脸、光线较暗、可见度低和人脸有遮挡 物存在的复杂情况下，本文方法的检测精度都高 于 AIZOO 方法.

结合实际情况的实验结果如图 8 所示. 图 8a 中, 在光照正常且面部无遮挡的多人同时存在的

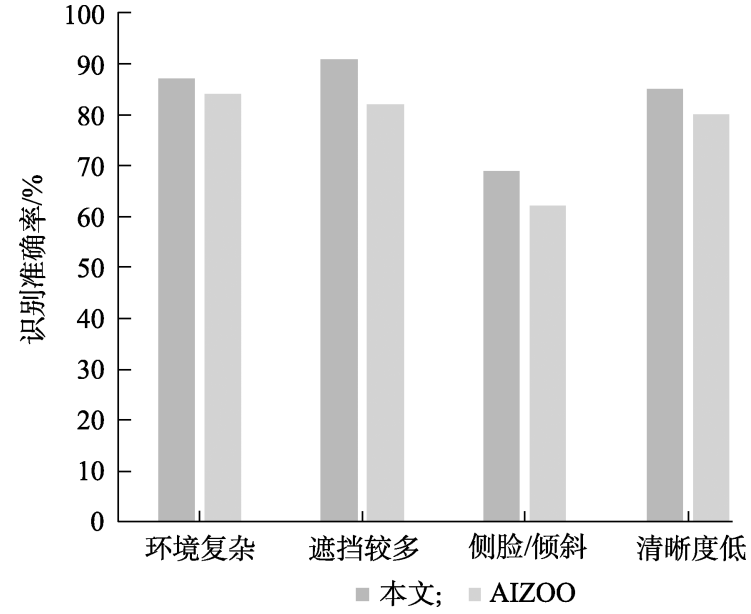

图 72 种方法实验结果

情况下，本文方法和 AIZOO 方法均实现了口罩佩 戴检测，正确地检测出了图片中的目标信息. 图 $8 \mathrm{~b}$ 中，在侧脸、人脸信息不完整的情况下，本文方 法对 2 个人的口罩佩戴信息均检测无误，而 AIZOO 方法检测结果不完整，对于图片中以侧脸 出现的人脸目标没有检测到其人脸信息, 说明针 对面部遮挡比较严重的目标, 本文方法的检测效 果相比 AIZOO 方法有一定的优势. 图 8c 中, 在侧 脸也存在光线不一的环境下, AIZOO 方法只检测 到了最右侧人脸信息, 且判断错误口罩佩戴状态, 而本文方法不仅成功地检测到完整的 2 个人脸信 息, 且对其口罩佩戴状态监测准确, 但最左侧人脸 信息由于光线太暗且人脸倾斜严重，无法获取人 脸关键点信息, 导致人脸信息未成功检测. 图 8d 中, 目标人物不仅戴了口罩, 还有帽子作为遮挡 物, 且背景较为杂乱, 这些条件都使识别人脸信息 的难度加大, 可以看出, 本文方法成功地识别了目 标口罩佩戴状态，而 AIZOO 方法检测失败.

实验结果表明，在人脸倾斜、背景环境复杂、 面部遮挡严重以及采光不足、室内环境昏暗等条件

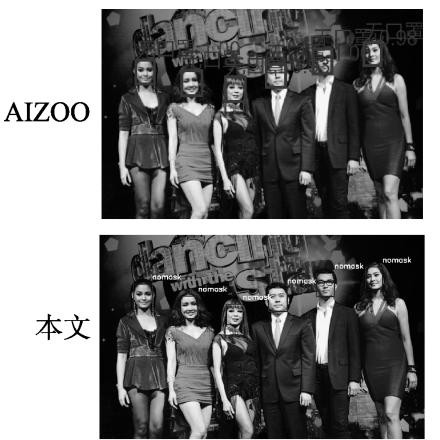

a. 正常情况

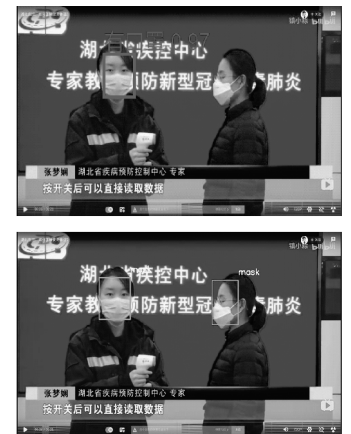

b. 面部倾斜

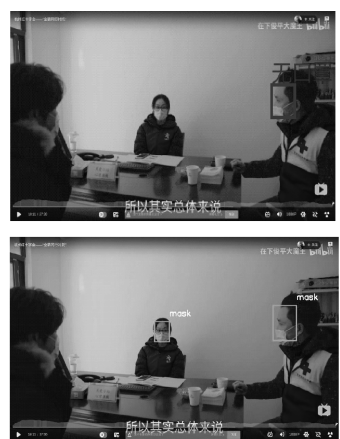

c. 光线昏暗

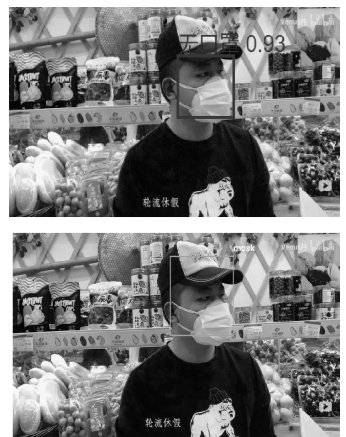

d. 面部遮挡

图 8 口罩佩戴检测效果图对比示例 
下，与 AIZOO 方法相比，本文方法不仅检测到的 人脸目标更多，且判断目标是否佩戴口罩时的检 测精度也高.

图 9 所示为本文方法检测不成功的示例.

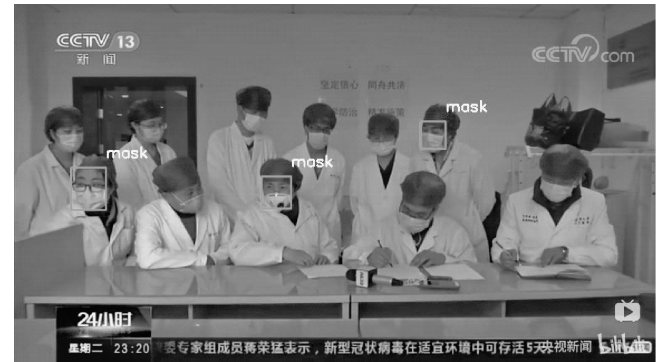

a. 光线较暗且面部遮挡严重

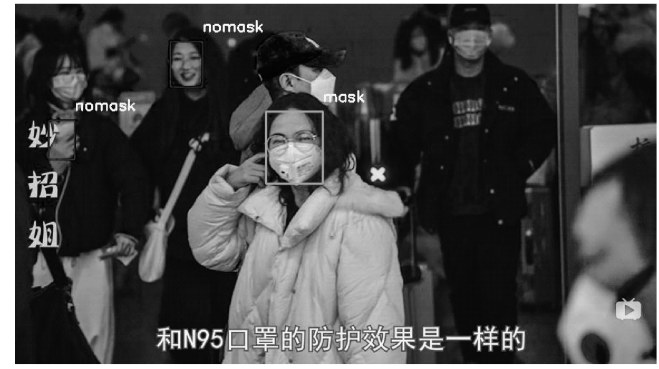

b. 光线不一, 背景虚化

图 9 检测不成功示例

\section{4 结 语}

针对复杂光照条件和人脸倾斜条件下的口罩 佩戴检测准确率低问题，本文提出一种基于轻量 级神经网络的口罩佩戴检测方法. 该方法利用难 样本挖掘尽可能多地获取更多准确的人脸特征, 由 MTCNN 对人脸进行检测定位, 生成人脸关键 点特征; 将关键点特征通过注意力机制处理后输 人 MobileNet, 对其进行检测和分类. 该方法快速 准确地定位脸部区域，之后对图片进行预处理、摆 正脸部等操作消除面部倾斜对口罩检测的影响. 实验结果表明，本文方法各项检测指标表现优于 其他口罩佩戴检测方法, 具有较强的鲁棒性和可 扩展性.

今后将从人脸遮挡严重以及光线较低等方向 开展工作. 如图 9 所示. 在脸部信息被大面积遮 挡、光线异常昏暗和图片清晰度较低的情况下，人 脸信息无法被成功识别，造成了口罩佩戴检测的 失败. 针对这种情况, 下一步将进行优化改进, 在 识别人脸信息时不仅要增加学习人体的头发和肢 体信息(以上半身为主), 也要重点学习眼睛的信息, 从而判别人脸的位置. 在目前的口罩佩戴检测工
作中，针对恶意遮挡(如使用非口罩物体对人脸的 口鼻进行遮挡)造成佩戴非口罩遮挡物逃过检测的 行为, 可以通过训练网络模型学习口罩类别信息, 以便更准确地判断遮挡物是否为口罩.

\section{参考文献(References):}

[1] Zhang K P, Zhang Z P, Li Z F, et al. Joint face detection and alignment using multitask cascaded convolutional networks[J]. IEEE Signal Processing Letters, 2016, 23(10): 1499-1503

[2] Jiang N, Yu W X, Tang S P, et al. A cascade detector for rapid face detection[C] //Proceedings of the 7th IEEE International Colloquium on Signal Processing and its Applications. Los Alamitios: IEEE Computer Society Press, 2011: 155-158

[3] Chen A, Xing H, Wang F Y. A facial expression recognition method using deep convolutional neural networks based on edge computing[J]. IEEE Access, 2020, 8: 49741-49751

[4] LeCun Y, Boser B, Denker J S, et al. Backpropagation applied to handwritten zip code recognition[J]. Neural Computation, 1989, 1(4): 541-551

[5] Liu W, Anguelov D, Erhan D, et al. SSD: single shot multibox detector[C] //Proceedings of European Conference on Computer Vision. Heidelberg: Springer, 2016: 21-37

[6] Szegedy C, Liu W, Jia Y Q, et al. Going deeper with convolutions[C] //Proceedings of the IEEE Conference on Computer Vision and Pattern Recognition. Los Alamitos: IEEE Computer Society Press, 2015: 1-9

[7] Ioffe S, Szegedy C. Batch normalization: accelerating deep network training by reducing internal covariate shift[J]. Journal of Machine Learning Research, 2015, 37: 448-456

[8] Krizhevsky A, Sutskever I, Hinton G E. ImageNet classification with deep convolutional neural networks[C] //Proceedings of the 25th International Conference on Advances in Neural Information Processing Systems. Red Hook: Curran Associates, 2012: 1097-1105

[9] Najibi M, Samangouei P, Chellappa R, et al. SSH: single stage headless face detector[C] //Proceedings of the IEEE International Conference on Computer Vision. Los Alamitos: IEEE Computer Society Press, 2017: 4885-4894

[10] Zhang S F, Zhu X Y, Lei Z, et al. $\mathrm{S}^{3} \mathrm{FD}$ : single shot scale-invariant face detector[C] //Proceedings of the IEEE International Conference on Computer Vision. Los Alamitos: IEEE Computer Society Press, 2017: 192-201

[11] Deng J K, Guo J, Zhou Y X, et al. RetinaFace: single-stage dense face localisation in the wild[OL]. [2020-09-01]. https://arxiv.org/pdf/1905.00641.pdf

[12] Tang X, Du D K, He Z, et al. PyramidBox: a context-assisted single shot face detector[C] //Proceedings of European Conference on Computer Vision. Heidelberg: Springer, 2018: 812-828

[13] Zhang S F, Wen L Y, Bian X, et al. Single-shot refinement neural network for object detection[C] //Proceedings of the IEEE Conference on Computer Vision and Pattern Recognition. Los Alamitos: IEEE Computer Society Press, 2018: 4203-4212

[14] Tian W X, Wang Z X, Shen H F, et al. Learning better features for face detection with feature fusion and segmentation super- 
vision[OL]. [2020-09-01]. https://arxiv.org/pdf/1811.08557.pdf

[15] Niu Zuodong, Qin Tao, Li Handong, et al. Improved algorithm of RetinaFace for natural scene mask wear detection[J]. Computer Engineering and Applications, 2020, 56(12): 1-7(in Chinese)

(牛作东, 覃涛, 李捍东, 等. 改进 RetinaFace 的自然场景口 罩佩戴检测算法 [J]. 计算机工程与应用, 2020, 56(12): 1-7)

[16] Li H C, Xiong P F, An J, et al. Pyramid attention network for semantic segmentation[C] //Proceedings of British Machine Vision Conference. Guildford: BMVA Press, 2018: 285

[17] Humphreys G W, Sui J. Attentional control and the self: the self-attention network (SAN)[J]. Cognitive Neuroscience, 2016, 7(1-4): 5-17

[18] Jiang H Z, Learned-Miller E. Face detection with the faster $\mathrm{R}-\mathrm{CNN}[\mathrm{C}] / /$ Proceedings of the IEEE International Conference on Automatic Face Gesture Recognition. Los Alamitos: IEEE Computer Society Press, 2017: 650-657

[19] Redmon J, Farhadi A. YOLOv3: an incremental improvement[OL]. [2020-09-01]. https://arxiv.org/pdf/1804.02767.pdf

[20] Shrivastava A, Gupta A, Girshick R. Training region-based object detectors with online hard example mining[C] //Proceedings of the IEEE Conference on Computer Vision \& Pattern Recognition. Los Alamitos: IEEE Computer Society Press, 2016: 761-769

[21] Howard A, Zhu M L, Chen B, et al. MobileNets: efficient convolutional neural networks for mobile vision applications[OL]. [2020-09-01]. https://arxiv.org/pdf/1704.04861.pdf

[22] Wan L, Eigen D, Fergus R, et al. End-to-end integration of a convolutional network, deformable parts model and non-maximum suppression[C] //Proceedings of the IEEE Conference on Computer Vision and Pattern Recognition. Los Alamitos: IEEE Computer Society Press, 2015: 851-859

[23] Tan Bin. Improvement of face detection MTCNN algorithm in classroom environment[D]. Chengdu: Xihua University, 2019(in Chinese)

(谭斌. 教室环境中人脸检测 MTCNN 算法的改进[D]. 成都:
西华大学, 2019)

[24] Ren S Q, He K M, Girshick R, et al. Faster R-CNN: towards real-time object detection with region proposal networks[J]. IEEE Transactions on Pattern Analysis and Machine Intelligence, 2017, 39(6): 1137-1149

[25] He Weixin, Deng Jianqiu, Liu Aidong, et al. Research on missile warehouse access control system based on MTCNN and RESNET face recognition[J]. Microcontrollers \& Embedded Systems, 2020, 20(4): 51-54(in Chinese)

(何伟金金, 邓建球, 刘爱东, 等. MTCNN 和 RESNET 的人脸 识别弹库门禁系统研究 [J]. 单片机与嵌人式系统应用, 2020, 20(4): 51-54)

[26] Yi Shuai, Zhu Jiansheng, Jing Hui. Face recognition technology applies in railway scene based on MTCNN face occlusion technology research[J]. Computer Simulation, 2020, 37(5): 96-99(in Chinese)

(衣帅, 朱建生, 景辉. 铁路刷脸场景下基于 MTCNN 的人脸 遮挡识别研究[J]. 计算机仿真, 2020, 37(5): 96-99)

[27] Simonyan K, Zisserman A. Very deep convolutional networks for large-scale image recognition[OL]. [2020-09-01]. https://arxiv.org/pdf/1409.1556.pdf

[28] He K M, Zhang X Y, Ren S Q, et al. Delving deep into rectifiers: surpassing human-level performance on ImageNet classification[C] //Proceedings of the IEEE Conference on Computer Vision and Pattern Recognition. Los Alamitos: IEEE Computer Society Press, 2015: 1026-1034

[29] Ranjan R, Patel V M, Chellappa R, et al. HyperFace: a deep multi-task learning framework for face detection, landmark localization, pose estimation, and gender recognition[J]. IEEE Transactions on Pattern Analysis and Machine Intelligence, 2019, 41(1): 121-135

[30] Wang Aoran, Liu Wei. Commodity recognition based on MobileNet model on TensorFlow platform[J]. Information \& Communications, 2018(12): 49-50(in Chinese) (王傲然, 刘玮. TensorFlow 平台上基于 MobileNet 模型的商 品识别[J]. 信息通信, 2018(12): 49-50) 